\title{
CONF- $9609319--1$ \\ Nuclear Structure Studies of Medium-Mass Nuclei Using \\ Large Ge Arrays
}

"The submitted manuscript has been authored by a contractor of the U.S. Government under contract No. DEAC05-960R22464. Accordingly, the U.S. Government retains a nonexclusive, royalty-free license to publish or reproduce the published form of this contribution, or allow others to do so, for U.S. Govemment purposes."

\author{
Cyrus Baktash
}

Physics Division, Oak Ridge National Laboratory, Oak Ridge, TN 37831-6371

\section{ABSTRACT}

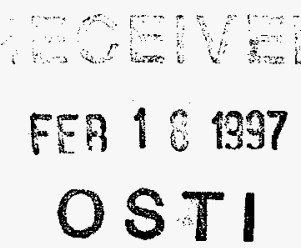

The advent of large Ge arrays and their ancillary detectors has greatly advanced spectroscopic studies of the medium-mass nuclei. These nuclei undergo rapid shape changes as a function of spin, excitation energy and particle number and, thus, provide a unique laboratory to test and refine a variety of theoretical models. Following a brief review of the physics motivation, some of the highlights of the experimental results obtained with the help of these powerful detector systems will be discussed. Among results presented here are the newly-discovered island of superdeformation in the A 80 mass region, and the high-spin band structures in the $N \sim Z$ nuclei. These band structures may be understood in the framework of the conventional cranking models, without the introduction of additional $\mathrm{T}=0$ neutron-proton pairing correlations.

\section{INTRODUCTION}

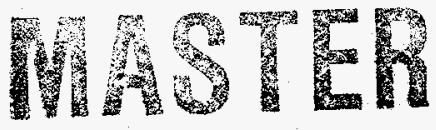

Medium-mass nuclei in the $A \approx 80$ region manifest a remarkable diversity of shapes and rapid changes of collectivity with particle number, angular momentum, and excitation energy. This distinction is rooted in the existence of large gaps in the neutron and proton single-particle energy spectra that appear at a variety of shapes and particle numbers. In the $N=Z$ nuclei where the protons and neutrons occupy the same orbitals, the shape driving effects of these deformed shell gaps are reinforced and give rise to a predicted rapid shape change from a spherical shape in ${ }^{56} \mathrm{Ni}$, to triaxial in ${ }^{64} \mathrm{Ge}$, possibly oblate in ${ }^{68} \mathrm{Se}$ and ${ }^{72} \mathrm{Kr}$, and very large prolate deformation in ${ }^{76} \mathrm{Sr}$ and ${ }^{80} \mathrm{Zr}$. In parallel, many of the nuclei with different numbers of neutrons and protons have a transitional character and are predicted to exhibit not only a rich pattern of shape coexistence (different shapes at comparable spins), but also to undergo shape transition (different shapes at different spins). Shape coexistence and shape transition in these nuclei result sometimes from the competition between the conflicting shell effects due to protons and neutrons, or sometimes from a delicate balance between the macroscopic and microscopic effects. Therefore, small differences in the parameters of the effective force, nuclear potential, or the macroscopic terms in the Hamiltonian can oftentimes result in largely different predictions for the structures of these nuclei. For example, the ground state band in ${ }^{84} \mathrm{Mo}$ has been predicted to be oblate by Moller and Nix [1981], prolate or oblate by Nazarewicz et al. [1985], and prolate by Petrovici et al. [1996]. Thus, experimental studies of the structure of these nuclei would provide a unique opportunity to test, compare and refine various theoretical models.

The $N=Z$ nuclei and their immediate neighbors are also a unique proving ground for the existence of the isoscalar $\mathrm{T}=0, \mathrm{~J}=1$ neutron-proton ( $n p$ ) pairing correlations. Although there is no unambiguous signature presently available for this new phase of pairing field, the high-spin band structures of the $N \sim Z$ nuclei may offer some clues as to when or if it can prevail over the familiar $\mathrm{T}=1$ pairing correlations.

In Section 2, we shall give a brief review of the experimental challenges that impede studies of the structures of the medium-mass nuclei and the recent advances in detector systems that have greatly helped to overcome them. This is followed by a survey of the systematics of the properties of the newly-discovered superdeformed nuclei in this region in Section 3, and new data on the high-spin band structures of several $T=1$ 


\section{DISCLAMMER}

Portions of this document may be illegible in electronic image products. Images are produced from the best available original document. 
nuclei in Section 4. Comparisons of the latter data set with theoretical calculations indicate no need to invoke $\mathrm{T}=0$ pairing correlations to interpret the band structures of the $N=Z+1$ and $N=Z+2$ nuclei studied so far.

\section{EXPERIMENTAL CHALLENGES AND THE ROLE OF NEW DETECTOR SYSTEMS}

Experimental studies of the high-spin band structures of the medium-mass nuclei have been impeded by several factors which are particular to this mass region. First, Because of the low Coulomb barrier and high excitation energy needed to populate the high-spin states, light charged-particle emission becomes dominant. As a result, the yields of the fusion-evaporation products fragments into a large number (typically 20 or more) of exit channels. Therefore, $\gamma$ rays from a variety of reaction products form a large background which can easily mask and interfere with those from the channels of interest. Second, due to the $A^{-5 / 3}$ scaling of the moment of inertia, the gamma rays associated with the medium-mass nuclei (and particularly those in the superdeformed bands) have high energies and are, thus, detected with poor photopeak efficiency. Third, reactions used to populate the high-spin states result in large velocities and angular spread of the recoiling nuclei which cause excessive Doppler broadening and poor energy resolution. This poses a particularly severe problem for the studies of reaction channels which involve emission of $\alpha$ particles.

The first of these difficulties may be alleviated by the use of large Ge detectors, while the second problem may be overcome in part by segmenting the Ge crystals. Therefore, the advent of the third generation of Gedetector arrays, namely the Eurogam [Beausang et al., 1988; Nolan, 1990] and Gammasphere, marks a great step forward in the spectroscopic studies of medium-mass nuclei. The Eurogam-II detector consists of 24 Clover and 30 large HPGe detectors with a total photopeak efficiency of $\sim 8 \%$. The Gammasphere is comprised of 110 HPGe detectors with a total photopeak efficiency of $\sim 10 \%$. Furthermore, approximately 70 of these detectors are electronically segmented into two halves to reduce their effective opening angle and, thus, the Doppler broadening of the gamma rays emitted from a moving source. As will be discussed in Sec. 3 , these developments paved the way for the observation of the first superdeformed (SD) band in this region using Eurogam-I array ${ }^{83} \mathrm{Sr}$ [Baktash et al., 1995a], and a systematic study of superdeformation and other high-spin band structures in A 80 nuclei using the Gammasphere array.

Another breakthrough was to combine the $4 \pi$ charged-particle detector Microball [Sarantites et al., 1996] with the Gammasphere array. Microball is an array of $95 \mathrm{CsI}(T t)$ elements with photodiode readout which provides for the detection of protons and $\alpha$ particles with an efficiency of $\sim 85$ and $\sim 75 \%$, respectively. Full detection of these particles and their energies helps in two ways. First, it serves to select and identify the exit channels of interest. Second, it allows the reconstruction of the recoil momenta which results in considerable improvements in the Doppler correction and, hence, gamma-ray energy resolution. This is best illustrated in Fig. 1, which compares gated $\gamma$-ray spectra for the yrast SD band in ${ }^{83} \mathrm{Sr}$ from two experiments. Panel (a) is the sum of several singles gates on the transitions in the band from the Eurogam-I experiment which produced the first example of superdeformation in this region [Baktash et al., 1995a]. Figure 1(b) was obtained by summing several double-gated spectra from a Gammsphere+Microball experiment which selected the ${ }^{83} \mathrm{Sr}$ channel by requiring that all of the four emitted protons be present in the data stream [LaFosse et al., 1995]. It shows a significant improvement in both the peak-to-background ratio (a factor of $\sim 8$ ) and the energy resolution of the gamma rays (a factor of $\sim 4$ ). Furthermore, the clean channel selection afforded by the Microball allowed detailed characterization of the band properties, including a better assignment of the spins and the point it intersects the normally-deformed (ND) yrast band [LaFosse et al., 1995].

The combination of Gammasphere and Microball arrays affords the selection of exit channels that may be produced with cross sections of $\geq 1 \mathrm{mb}$. To further lower the threshold for the selection and identification of very weakly populated exit channels, the above system has been augmented by 15 liquid scintillator neutron detectors with a combined trigger efficiency of $\sim 5 \%$. This combination of the arrays of gamma, chargedparticle and neutron detectors has been successfully used to study the $N=Z$ nuclei ${ }^{52} \mathrm{Fe}$ [Abdelrazek et al., 

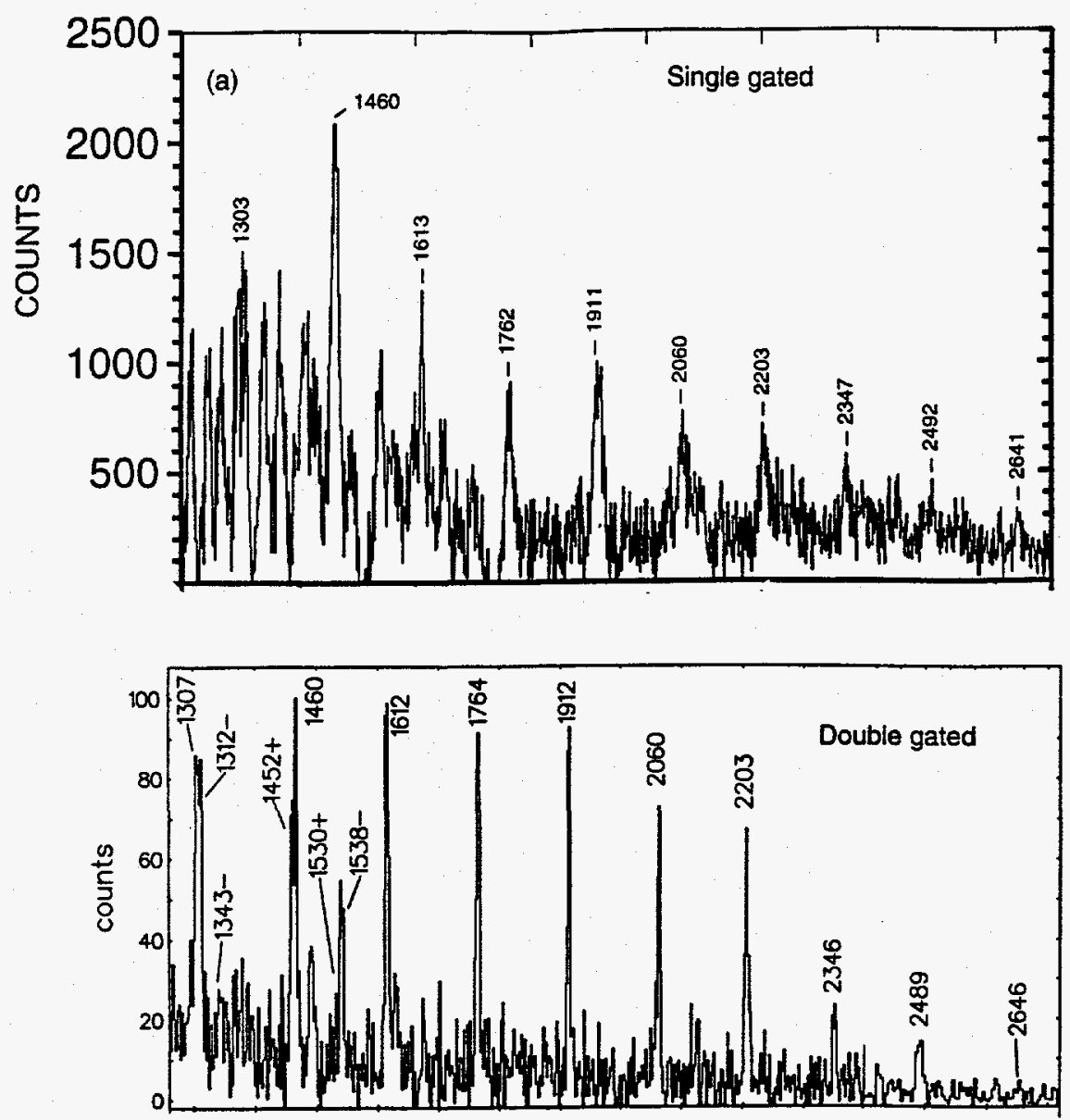

Figure 1: Comparison of the gated spectra of the SD band in ${ }^{83} \mathrm{Sr}$ obtained in two experiments [Baktash et al., 1995a; LaFosse et al, 1995] (see text for explanation).

1996), ${ }^{54} \mathrm{Co},{ }^{58} \mathrm{Cu}$, and the ${ }^{55} \mathrm{Ni}-{ }^{55} \mathrm{Co}$ mirror pair [Rudolph et al., 1996c] using the reaction of ${ }^{36} \mathrm{Ar}+{ }^{28} \mathrm{Si}$ at a beam energy of $136 \mathrm{MeV}$. The selectivity of this system is illustrated in Fig. 2 which shows how an inclusive spectrum associated with all channels emitting $3 p$ (middle panel) may be further filtered by requiring the presence of an additional neutron (top panel) or $\alpha$ (bottom panel). The lowest cross section measured in this experiment was that of the ${ }^{55} \mathrm{Ni}$, estimated to be $\sim 40 \mu \mathrm{b}$.

Spectroscopy of nuclei produced with cross sections of 1-100 $\mu \mathrm{b}$ can greatly benefit from the use of recoil mass separators (RMS). For example, the combination of the Daresbury recoil separator [James et al., 1988] and the POLYTESSA Ge array with a photopeak efficiency of $\sim 1 \%$ [Simpson et al., 1988] was used for the pioneering studies of the $N=Z$ nuclei from ${ }^{64} \mathrm{Ge}(\sigma \sim 640 \mu \mathrm{b})$ [Ennis et al., 1991] to ${ }^{84} \mathrm{Mo}(\sigma \sim 7 \mu \mathrm{b})$ [Gelletly et al., 1991]. Extensions of these studies toward the doubly-magic self-conjugate nucleus ${ }^{100} \mathrm{Sn}$ requires radioactive ion beams (RIB) and an even more powerful set of detector systems.

One such system is being assembled at the Holifield Radioactive Ion Beam Facility at ORNL, where accelerated RIB will be used to investigate the structure of nuclei near the proton-drip line. This system consists of a state-of-the-art RMS [Cormier et al., 1983; Mantica et al., 1995; Gross et al., 1996a], and a variety of ancillary detectors at the target position and the focal plane to provide the required selectivity. The RMS along with a position sensitive avalanche counter and an ionization chamber, both located at the focal plane, will provide excellent mass selection (a mass resolution of 1 part in 450 for symmetric reactions) and $Z$ information up to $Z \sim 40$. For $Z>40$, a Hybrid $4 \pi$ charged-particle detector and an array of neutron detectors at the target position will provide supplementary atomic number information to separate the isobars. 


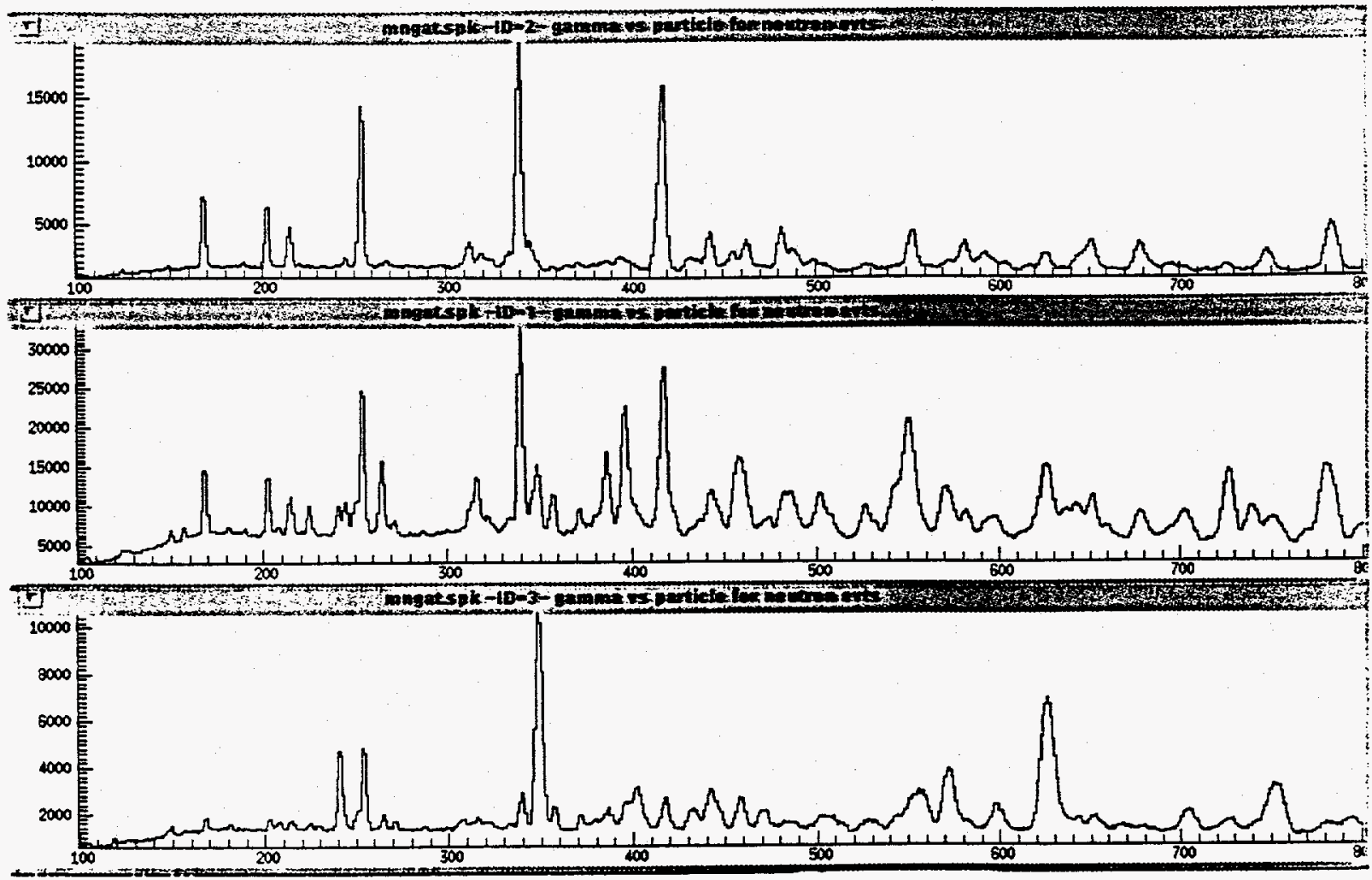

Figure 2: Three $\gamma$-ray spectra gated with at least 3 protons from the ${ }^{36} \mathrm{Ar}+{ }^{28} \mathrm{Si}$ reaction at $136 \mathrm{MeV}$ : (Top) gated with $3 p n$ leading to ${ }^{60} \mathrm{Cu}$; (Middle) an inclusive spectrum of $3 p$; (Bottom) gated with $3 p \alpha$ leading to ${ }^{57} \mathrm{Co}$.

The charged-particle detector consists of $80 \mathrm{CsI}$ detectors (similar to the Microball elements) for angles greater than $25 \mathrm{deg}$, and fast detectors to cover laboratory angles of 7-25 deg. The geometry of these detectors, schematically shown in Fig. 3, is designed to reduce the exposure of the Ge and CsI detectors to the background of the $511 \mathrm{keV} \gamma$ rays and the positrons which result from the decay of the Rutherford-scattered radioactive ions stopped in the wall chambers or the absorbers. The opening angle of the forward funnel in the target chamber ( $25 \mathrm{deg}$ ) has been optimized using Monte Carlo simulations. Similarly, the background of radioactivity requires that the forward charged-particle detectors be placed at a large distance $(\sim 30 \mathrm{~cm})$ from the target, and be fast enough to tolerate count rates of up to $20,000 \mathrm{~Hz}$ due to the positrons. Plastic phoswich or strip $\mathrm{Si}$ detectors are being investigated as possible candidates.

The Ge array in this setup consists of 12 elements of the existing ORNL Compton Suppressed Spectrometer (CSS), augmented by up to 11 Clover detectors. All Ge detectors are equipped with BGO Compton suppression shields. The CSS detectors are placed at a distance of $23 \mathrm{~cm}$ from the target and provide a combined photopeak efficiency of $\sim 0.7 \%$. Each Clover detector consists of four HPGe crystals with $25 \%$ relative efficiency. In the so called addback mode, where the signals from all four elements are added together, each Clover forms a $\sim 8 \times 8 \times 8 \mathrm{~cm}^{3}$ composite detector with a relative efficiency of $150 \%$. In 10 detectors, each of the four elements are electronically segmented into two halves to reduce the angular opening and, hence, the Doppler broadening effects. This provides a positional information of $-2 \mathrm{~cm}$ which, at a distance of $15 \mathrm{~cm}$ from the target, translates to a FWHM resolution of about $7 \mathrm{keV}$ for a $1332 \mathrm{keV}$ gamma ray emitted from a source moving with a velocity of $v / c=0.05$. This is a better resolution than that obtained with a conventional Clover detector or a segmented Gammasphere detector placed $23 \mathrm{~cm}$ from the target. The 11 Clover detectors in this setup are placed at a distance of $18 \mathrm{~cm}$ from target and have a combined photopeak 


\section{Target Position Detectors}

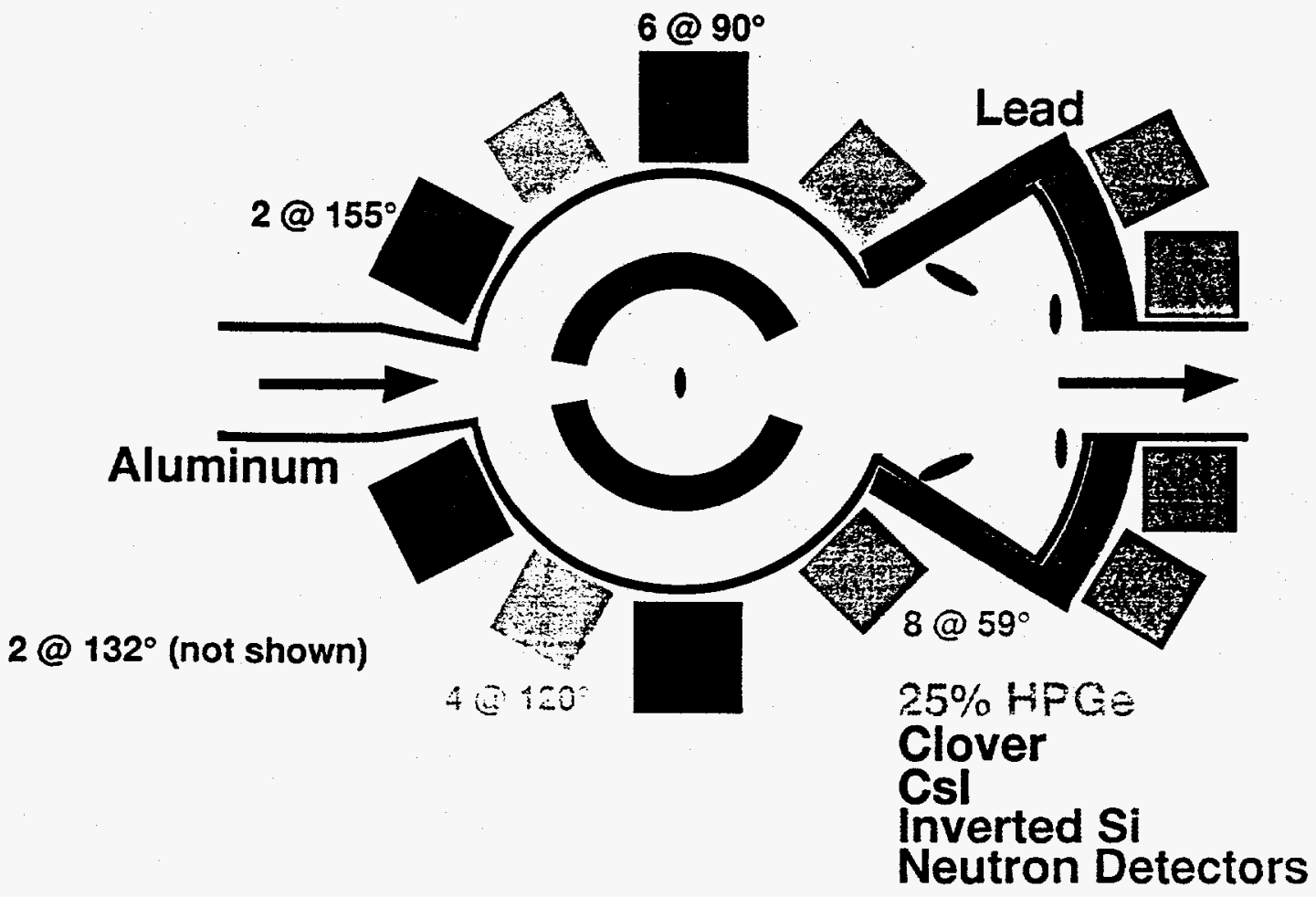

Figure 3: Schematics of the geometry of the Ge array, charged-particle, and neutron detectors at the target-position of the RMS at HRIBF.

efficiency of $3.3 \%$. Therefore, the total photopeak efficiency of this system is $4 \%$, which is comparable with that of the Ga.Sp. array at Legnaro.

\section{SUPERDEFORMATION IN THE MASS A 80 REGION}

Superdeformation was first discovered in the actinide fission isomers [Polikanov et al., 1962], and was explained as resulting from a secondary minimum at superdeformed (SD) shapes [Strutinsky, 1967, 1968]. In the actinide region, the sum of the macroscopic liquid drop term, and the microscopic shell correction to the total energy gives rise to a second minimum at SD even at very low spins. However, with the weakening of the Coulomb force in lighter systems, SD minima are stabilized only at high spins and for certain nuclei close to SD magic numbers. Studies of these high-spin SD structures, and especially their mass dependence, provide an important insight into the competition between the microscopic and macroscopic effects, the role of the odd-time terms in the Hamiltonian, as well as certain aspects of the effective interactions such as the spinorbit force.

The first high-spin SD band was observed in ${ }^{152}$ Dy by Twin et al. in 1986, and was followed the by discovery of another island of superdeformation around ${ }^{192} \mathrm{Hg}$ [Moore et al., 1989]. These findings were in excellent agreement with the results of the earlier calculations which had predicted the existence of superdeformed magic particle numbers $N, Z \sim 44,64,86$, and 116 [Ragnarsson et al., 1978; Nazarewicz et al., 1985]. Encouraged by these experimental successes, numerous searches were undertaken to verify the existence of the predicted superdeformed shell gaps near particle number 44 , as shown in Fig. 4 . However, due to the experimental difficulties discussed in Sec. 2, these efforts were not successful until recently. Thus, the observation of the first SD in ${ }^{83} \mathrm{Sr}$ [Baktash et al., 1995a] had to await the advent of arrays of large $\mathrm{Ge}$ detectors such as Eurogam-I. 


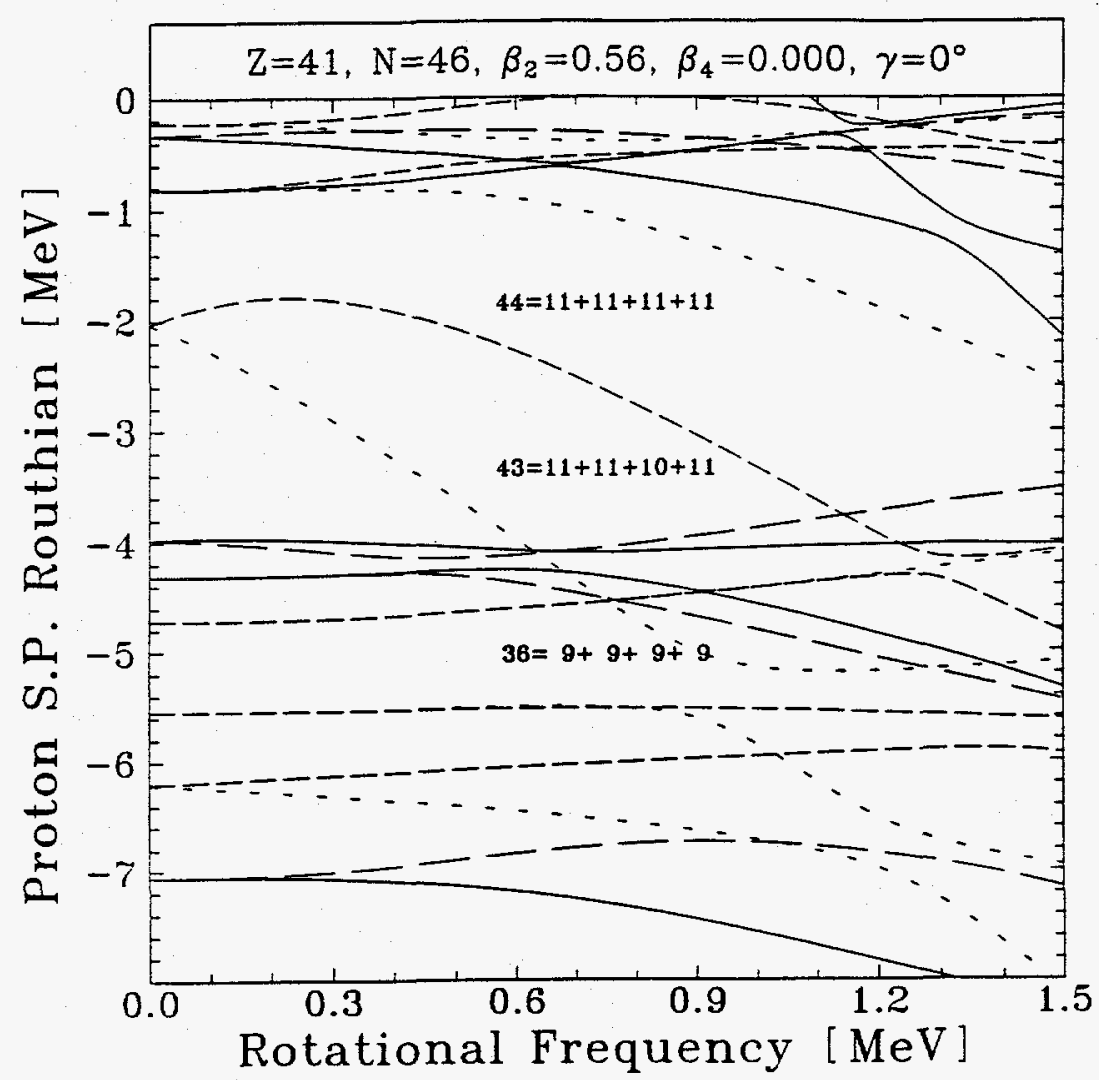

Figure 4: Routhians of the single-particle protons as a function of rotational frequency for a deformation parameter $\beta_{2}=0.56$. Note the large shell gap at particle number 44 .

Thanks to the development of large Ge arrays and $4 \pi$ charged-particle detectors discussed in the previous section, more than $20 \mathrm{SD}$ bands in ten medium-mass nuclei have been found in the past two years. They include multiple bands in ${ }^{80,81,82} \mathrm{Sr}$ [Devlin et al., 1996; Cristancho et al., 1995; Smith et al., 1995], ${ }^{83,84} \mathrm{Y}$ [Jin et al., 1996a], ${ }^{83} \mathrm{Zr}$ [Rudolph et al., 1996a], ${ }^{87} \mathrm{Nb}$ [LaFosse et al., 1997], as well as the yrast SD bands in ${ }^{83} \mathrm{Sr}$ [Baktash et al., 1995a; LaFosse et al, 1995], ${ }^{82} \mathrm{Y}$ [Dagnall et al., 1995], and ${ }^{84} \mathrm{Zr}$ [Jin et al., 1995]. In the following, we review the general properties of these bands and compare their characteristics with those of the SD bands in the heavier mass resigns.

\subsection{Experimental observables and their systematics}

Some of the general properties of SD bands that may be experimentally studied to test various theoretical models are: the $J^{(2)}$ moments of inertia and their variations with rotational frequency, transition quadrupole moments $\left(Q_{1}\right)$, measurements of the g-factors either directly or indirectly from the $B(M 1) / B(E 2)$ ratios, and identification of the excited bands and their relative spins and excitation energies with respect to the yrast SD band. To varying degrees, all these observables are sensitive to, and help identify the single-particle configurations of the SD bands. Other observables which are also relevant to this question are the relative alignments of different SD bands, either in the same nucleus or in neighboring isotopes and isotones. This quantity may be simply deduced from the $\gamma$-ray energies, and determines the fractional change in the $J^{(2)}$ of the pair of bands as discussed by Baktash et al. [1995b].

The feeding pattern of the SD bands and their variations (if any) with reaction may shed some light on the fusion-evaporation reaction mechanism, and factors which limit both population and retention of the high partial waves in compound nuclei. The decay pattern is related to the quantum-mechanical tunneling through the barrier which separates the SD and ND states, and depends on such factors as the barrier height, level density, and pairing interaction. 
The first SD in this mass region was observed by Baktash et al. [1995a] using the Eurogam-I array and the ${ }^{56} \mathrm{Fe}\left({ }^{30} \mathrm{Si}, 2 p n\right){ }^{83} \mathrm{Sr}$ reaction at a beam energy of $128 \mathrm{MeV}$. This and similar reactions, which form compound nuclei at $\sim 70 \mathrm{MeV}$ excitation energy and $>50 \hbar$ angular momentum, have been found to be optimal for the population of SD bands in the $4 p, 3 p, 2 p n$, and in particular the $\alpha 2 p$ and $\alpha p n$ exit channels. Our studies indicate that emission of alpha particles, which originate from the highest angular momentum states in these compound nuclei, is very effective in populating the high-spin states and SD bands in medium-mass nuclei (see, e.g., data on $T_{z}=1$ nuclei by Rudolph et al. [1996b] presented in Sec. 4). It should be noted that since such a relatively high excitation energy corresponds to the maximum production of the four-particle emission channels, the above three-particle channels are populated with only modest cross sections.

Similar to the SD bands in heavier nuclei, these bands are fed over a range of several transitions and generally reach a saturation intensity of $<2 \%$ of the total. The exceptions are the yrast bands in ${ }^{83} \mathrm{Zr}$ [Rudolph et al., 1996a] and ${ }^{84} \mathrm{Zr}$ [Jin et al., 1995], which are populated with intensities of more than 5\% and $4 \%$, respectively. Both of these values are about a factor of 2 larger than the intensities of the yrast SD bands in their $\mathrm{Y}$ isotones that were populated via the $3 p$ channel in the same reactions. In the case of ${ }^{84} \mathrm{Zr}$, significant differences were observed in the feeding saturation point when the band was populated by two different reactions, namely $2 p n$ and $\alpha 2 p$ [Jin et al., 1995]. This is the only known example of this effect and points to an important new factor that influences the population of SD bands. Also, the common assumption that the half-intensity point (i.e., the level which receives $50 \%$ of the total intensity of the band) coincides with the point where SD bands become yrast is called into question due to the fact that this point was observed to vary with reaction.

The decay of these bands is sudden and is completed within a narrow range of one to three transitions. As is the case in the heavier nuclei, these SD bands decay statistically to several ND bands of both parities. From the intensities of the feeding-out (of the SD) and feeding in (to the ND) transitions, one can estimate the approximate spins of the SD bands. The spins of the lowest-lying states have been estimated to be approximately $15 \hbar$ in the $N=43$ nuclei ${ }^{81} \mathrm{Sr}$ [Cristancho et al., 1995] and ${ }^{83} \mathrm{Zr}$ [Rudolph et al., 1996a], and close to $20 \hbar$ in the more neutron-rich nuclei ${ }^{82,83} \mathrm{Sr}$ [Smith et al., 1995; LaFosse et al., 1995] and ${ }^{84} \mathrm{Zr}$ [Jin et al., 1995]. Many of these bands extend to a maximum spin of $\sim 40 \hbar$. Assuming that the yrast SD and ND bands cross each other at a point where their side feeding intensities are nearly equal, it is estimated that these SD bands become yrast at $\sim 30 \hbar$. Interestingly, this spin corresponds to the onset of band termination that has long been predicted [Nazarewicz et al., 1985] to occur in many of the ND bands in this region. Band termination was recently observed in several nuclei such as ${ }^{83} \mathrm{Sr}$ [Cullen et al, 1994], ${ }^{83} \mathrm{Y}$ [Jin et al., 1996b], and ${ }^{84} \mathrm{Zr}$ [Jin et al., 1995], as evidenced in their rapidly decreasing $J^{(2)}$ (see Fig. 5a) and $\mathrm{Q}_{\mathrm{t}}$ values [Yu et al., 1996].

\subsubsection{Systematics of the moments of inertia and identical bands}

A very distinctive feature of these bands is the high energies of their gamma rays which typically range from about 1300 to more than $2600 \mathrm{keV}$. This may be compared with a maximum gamma-ray energy of approximately 700 and $1500 \mathrm{keV}$ for the SD bands in the A 190 and 150 regions, respectively [Han and Wu, 1992]. The energy differences of the consecutive gamma rays in the SD bands of the A 80 nuclei are approximately $150 \mathrm{keV}$, which imply a dynamical moment of inertia $J^{(2)}-27 \hbar^{2} / \mathrm{MeV}$, corresponding to that of a rigid rotor with a quadrupole deformation of $\beta_{2} \sim 0.5$, or major-to-minor axis ratio of $2: 1$. 
Figure $5 \mathrm{~b}$ shows a plot of the $J^{(2)}$ versus rotational frequency $(\omega)$ for the $\mathrm{SD}$ band in ${ }^{83} \mathrm{Sr}$, along with those in ${ }^{240} \mathrm{Pu}, \quad{ }^{192} \mathrm{Hg}$, and ${ }^{152} \mathrm{Dy}$, which represent typical SD bands in their respective mass regions [Han and $\mathrm{Wu}$, 1992]. To better demonstrate the similarity of these bands, all moments of inertia were scaled by $\mathrm{A}^{-5 / 3}$ to factor out the average mass-number dependence. Apart from the A 190 region, where the values rise continuously with $\omega$, the scaled moments of inertia of the other three SD bands have comparable values and are nearly constant as a function of $\omega$. The band in ${ }^{83} \mathrm{Sr}$ extends to a maximum rotational frequency of about $1.3 \mathrm{MeV}$, nearly twice as high as the frequencies encountered in the $A \sim 150$ region. Thus, the SD bands in the A 80 region represent the highest collective rotational frequency observed so far.

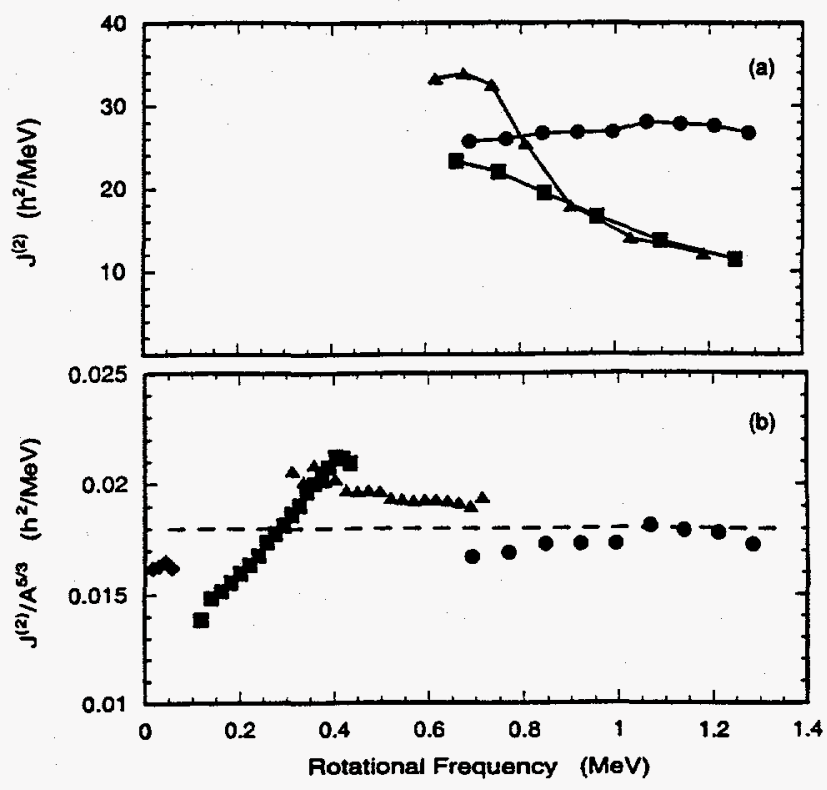

Figure 5: Plots of scaled $J^{(2)}$ vs. rotational frequency. (a) The SD band in ${ }^{83} \mathrm{Sr}$ (circles) and two ND bands in ${ }^{83} \mathrm{Y}$ (squares) and ${ }^{82} \mathrm{Sr}$ (triangles).

(b) SD bands in ${ }^{240} \mathrm{Pu}$ (diamonds), ${ }^{192} \mathrm{Hg}$ (squares), ${ }^{152} \mathrm{Dy}$ (triangles), and ${ }^{83} \mathrm{Sr}$ (circles). The dashed line shows the corresponding plot for a SD rigid rotor with $\beta_{z}=0.5$.

Figure 6 shows the systematics of the $J^{(2)}$ of the SD bands in ten nuclei in this region. Apart from a few cases (e.g., ${ }^{81} \mathrm{Sr}$ or ${ }^{83} \mathrm{Zr}$ ) where discontinuities due to band crossing are present, the majority of the $J^{(2)}$ values fall in the range of $-25-30 \hbar^{2} / \mathrm{MeV}$.

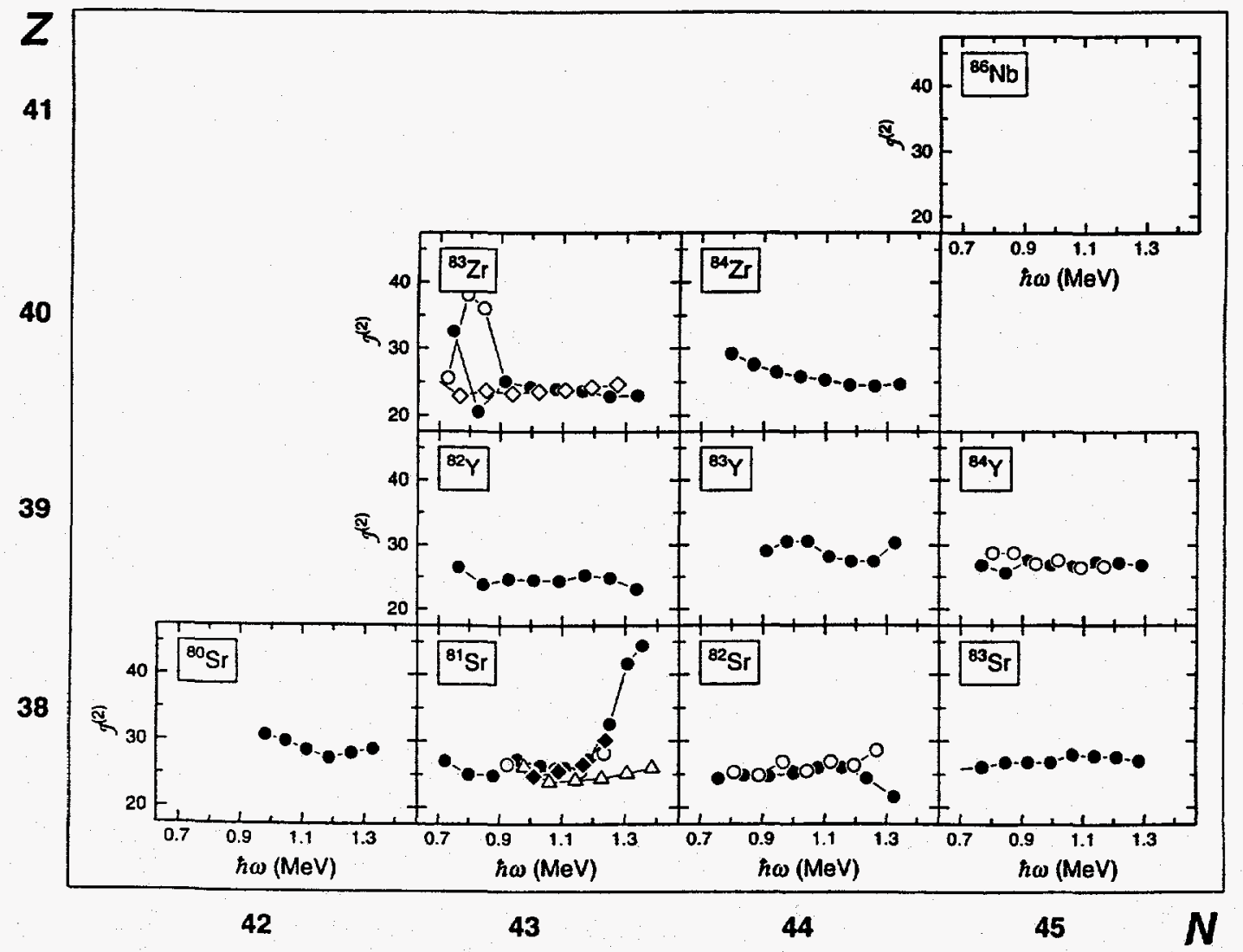

Figure 6: Systematics of the $f^{(2)}$ moments of inertia for the SD bands in the A 80 region. 
A convenient way to compare the $J^{(2)}$ values of these bands is to calculate the fractional changes, $\Delta J^{(2)} / J^{(2)}$, of pairs of bands as described by Baktash et al. [1995b]. A histogram of the fractional changes in moments of inertia is shown in Fig. 7, which compares all bands vs. all others (solid), and all bands vs. the yrast bands in their adjacent A+1 nuclei (dashed). In the latter category, nearly $16 \%$ of the bands are identical, as compared with $\sim 12 \%$ and $4 \%$ for the SD bands in the $A \sim 150$ and 190 regions, respectively [de France et al., 1996]. Special among these identical bands are two pairs of twinned bands (i.e., bands with similar gamma-ray energies) in ${ }^{82} \mathrm{Sr}$ vs. ${ }^{83} \mathrm{Y}$ and ${ }^{83} \mathrm{Sr}$ vs. ${ }^{84} \mathrm{Y}$ [Jin et al., 1996a], which imply the existence of a single-particle routhian with a slope of $-0.5 \hbar$ at $Z=39$. As discussed in Sec. 3.3, this information may be used to test theoretical models and to constraint their parameters.
Mass 80 SD bands

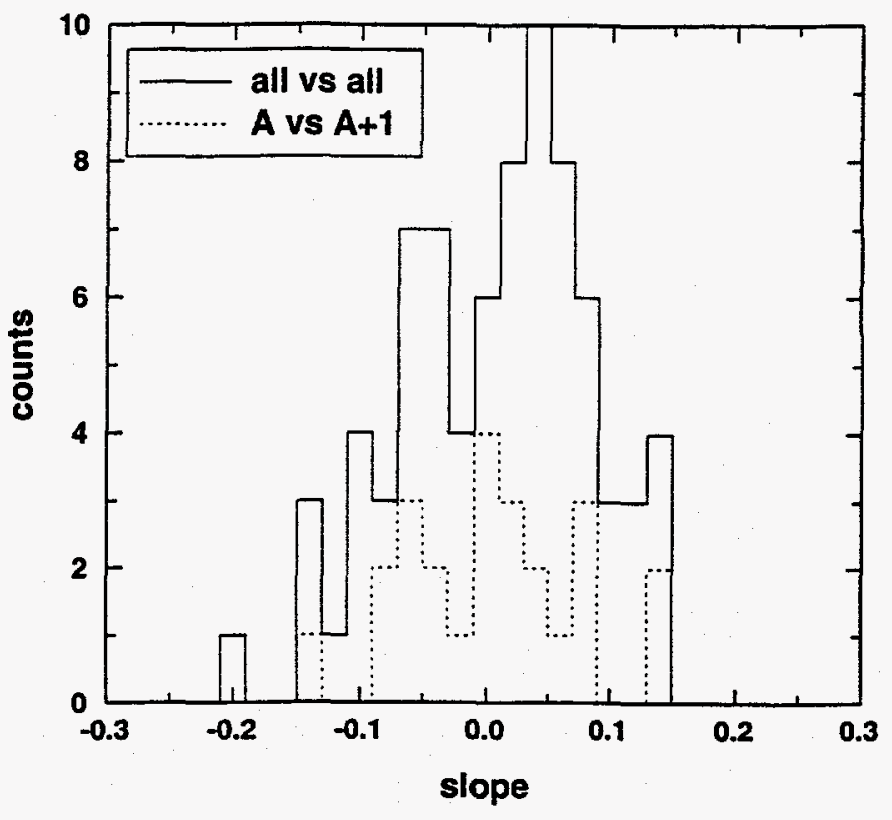

Figure 7: Histogram of frequency distributions of the fractional changes $\Delta J^{(2)} / J^{(2)}$ of pairs of SD bands in the $\mathrm{A} \sim 80$ region (see text).

Other experimental observations which may be used for the same purpose are the rotational frequencies where band crossings take place and their interaction strengths. A particularly interesting case is the band crossing encountered in ${ }^{87} \mathrm{Nb}$ [LaFosse et al., 1997]. The observed interband transitions and their branching ratios indicate that the crossing between the neutron [420]1/2 and [413]7/2 orbitals is responsible for this interaction. This fact may be used to infer the relative position of these orbitals. Furthermore, a second band interaction observed in only one of the two bands may be interpreted as due to the crossing with the neutron $i_{13 / 2}$ orbital which originates from the $\mathrm{N}=6$ shell. This is the first evidence for the involvement of a "superintruder" $(\Delta N=3)$ in the configuration of a SD structure and represents a Coriolis interaction $(\hbar \omega j \sim 8.25 \mathrm{MeV})$ which is about 35\% larger than anything observed up to now. What makes this unique situation possible is a combination of the large deformation and extremely high rotational frequency which lowers the energy of this superintruder orbital.

\subsubsection{Transition quadrupole moments and deformations}

Because of both the large B(E2) values and high energies of the intraband transitions, the SD states have very short lifetimes. For example, the sum of the feeding and decay times for the top few states is typically a few $\mathrm{fs}$, while the whole band decays in about $50 \mathrm{fs}$ which is comparable to the transit times of the recoils through a thin $\left(-400 \mu \mathrm{g} / \mathrm{cm}^{2}\right)$ target foil. Therefore, the traditional DSAM technique with backed targets is too slow to be useful for the measurements of the lifetimes of these states. On the other hand, since the recoils do experience detectable reductions in their velocities as they traverse through the thin target foil, their velocity profiles provide a useful clock to measure the decay times. Thus, by measuring the centroids of the Doppler-shifted gamma rays in the forward and backward Ge detectors, one can obtain average fractional shifts $\mathrm{F}(\tau)=\left\langle\nu(\tau) / v_{0}>\right.$ which can be compared with the calculated values to obtain the $\mathrm{Q}$, of the band [Cederwall et al., 1995]. $(v(\tau)$ is the instantaneous recoil velocity of the nucleus as deduced from the observed centroid shifts and $v_{0}$ is the maximum recoil velocity in the experiment.) This method was successfully used to measure an average $Q_{t}$ of $5.2 \pm 0.8 \mathrm{eb}$ for the SD band in ${ }^{84} \mathrm{Zr}$ [Jin et al., 1995], which corresponds to a B(E2) strength of $440 \mathrm{~W}$.u. or a quadrupole deformation of $\beta_{2}=0.53$ for an axially symmetric prolate shape. This and other $Q_{1}$ measurements clearly establish the SD character of these bands. 
An important question to be addressed by future $Q_{t}$ measurements is what are the particle numbers that give rise to the largest SD gaps. The present data (which are admittedly of poor statistical quality) indicate a gradual increase of $\mathrm{Q}_{\mathrm{t}}$ for the $\mathrm{Sr}$ isotopes from $N=42$ toward $N=44$ [Devlin et al., 1996; Cristancho et al., 1995; Yu et al., 1996], and a larger $\mathrm{Q}_{\text {, for }}{ }^{84} \mathrm{Zr}$ than either ${ }^{82} \mathrm{Sr}$ or ${ }^{83} \mathrm{Y}$ for the $N=44$ isotones [Jin et al., 1995; Yu et al., 1996; Jin et al., 1996a]. The latter trend is shown in Fig. 8, which is a plot of the $F(\tau)$ vs. $\gamma$-ray energy for the transitions from the ND states in ${ }^{82} \mathrm{Sr}$, and from the SD levels in ${ }^{82} \mathrm{Sr}$ and ${ }^{84} \mathrm{Zr}$. It is worth noting that the data points for the longer-lived ND states cluster around the dashed line at $v / v_{0}$ $=0.94$, which corresponds to the velocity of the recoils after they have left the target. Nearly all the data points for ${ }^{84} \mathrm{Zr}$ band lie above those for ${ }^{82} \mathrm{Sr}$, indicating shorter lifetimes for the states in the former band.

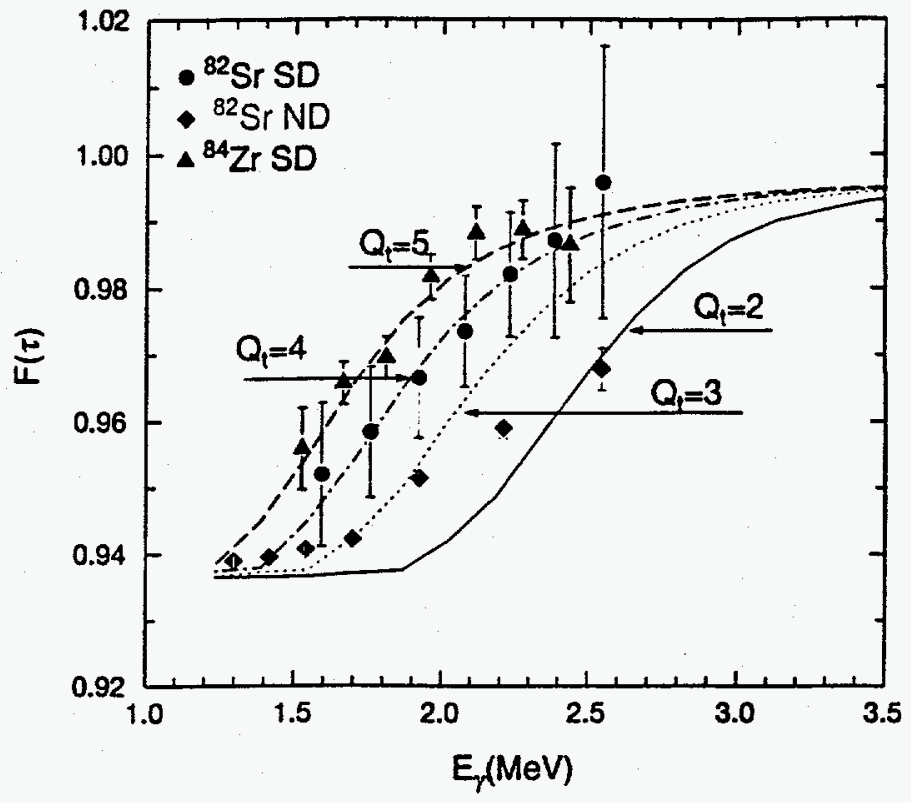

Figure 8: Plots of $F(\tau)$ curves vs. $\gamma$-ray energies for the SD and some ND states in ${ }^{82} \mathrm{Sr}$ and ${ }^{84} \mathrm{Zr}$ [Yu et al., 1996].

Although $Q_{1}$ values are sensitive to other variables (e.g., the number of the high- $j$ intruder orbitals involved in their configurations [Satula et al., 1996a]), these preliminary results suggest that $N=44$ and $Z=40$ are optimal particle numbers for the formation of SD structures. As noted in Sec. 3.1.1, the SD bands in ${ }^{83.84} \mathrm{Zr}$ are populated twice as strongly as other SD bands studied so far which also supports the idea that $Z=40$ is an optimal proton number. Nevertheless, these early conclusions await confirmation by future precision measurements which would populate these bands under identical conditions. For example, the bands in the ${ }^{82} \mathrm{Sr},{ }^{83} \mathrm{Y}$, and ${ }^{84} \mathrm{Zr}(N=44)$ isotones may be studied via $(\alpha 2 \mathrm{p})$ reactions induced by the ${ }^{30} \mathrm{Si}$, ${ }^{31} \mathrm{P}$, and ${ }^{32} \mathrm{~S}$ beams on the same ${ }^{58} \mathrm{Ni}$ target and at beam energies that produce the same entry-state distributions in the corresponding compound nuclei. Similarly, the $N=44$ to $46 \mathrm{Sr}$ isotopes may be produced by the reactions of ${ }^{28.29 .30} \mathrm{Si}$ beams on the same ${ }^{60} \mathrm{Ni}$ target. A comparison of the feeding patterns, total intensities, and the $\mathrm{Q}_{\text {, }}$ values of these bands populated under similar conditions would provide valuable insight into the relative contributions of the $N \sim 45$ and $Z \sim 39$ orbitals to their structures.

\subsubsection{Forking and unusual decays}

One of the SD bands in each of the ${ }^{83} \mathrm{Zr}$ [Rudolph et al., 1996a] and ${ }^{87} \mathrm{Nb}$ [LaFosse et al., 1997] nuclei fork into two branches near the bottom of the cascade. Their $J^{(2)}$ moments of inertia indicate that the close-lying levels in the two branches interact with each other. Evidence has also been presented that two levels in one of the bands in ${ }^{81} \mathrm{Sr}$ [Cristancho et al., 1995] interact with two other states which lie only $\sim 10 \mathrm{keV}$ away. More interestingly, in the case of the $N=83 \mathrm{Sr}$ and $\mathrm{Zr}$ isotones, the routhians of the bands curve down and reapproach those of the ND states, allowing for their mutual interaction. (This unusual situation cannot be avoided for any reasonable values of the bandhead energies which are consistent with the side feeding intensities.) A similar situations has not been encountered in the heavier SD regions. The proximity of these SD bands to the ND bands may provide a favorable condition for the observation of the direct linking transitions. 
Many of the observed properties of the SD bands in the A 80 region are in good agreement with the predictions made using the cranked Strutinsky method (see, e.g., Baktash et al., [1995a]; Cristancho et al. [1995]; Jin et al. [1995]; LaFosse et al., [1995]; and Smith et al., [1995] for comparisons of $J^{(2)}, \beta_{2}$, and the calculated low-lying SD bands). These calculations incorporate the Woods-Saxon potential for the microscopic part and the Yukawa-plus-exponential mass formula for the macroscopic part of the total energy. For a given configuration, the equilibrium deformations at fixed values of $\omega$ were determined by minimizing the total energy at each $\left(\beta_{2}, \gamma\right)$ grid point with respect to the hexadecapole deformation. Pairing correlations were neglected, as they are expected to play a minor role at high-spins in the A 80 mass region. Details of the calculational procedure and technique are given in papers by Nazarewicz et al., [1985] and Dudek et al. [1987].

As seen in Fig. 9, these calculations predict a number of low-lying SD structures that have deformations ranging from $\beta_{2} \sim 0.45$ to 0.55 , depending on how many $h_{11 / 2}$ intruder orbitals are involved in their configurations (see, e.g., Refs. [Baktash et al., 1995a; Cristancho et al. 1995; Smith et al., 1995]). At high spins, they generally have one proton in the $h_{11 / 2}$ orbital, symbolized as $\pi 5^{1}$. The neutron configurations of the yrast SD bands contain one to three $h_{11 / 2}$ orbitals for $N=43-45$, respectively. Therefore, a gradual increase in deformation is expected in going from $N=42$ in ${ }^{80} \mathrm{Sr}$ to $N=45$ in ${ }^{83} \mathrm{Sr}$, in good agreement with the preliminary $Q_{t}$ measurements.

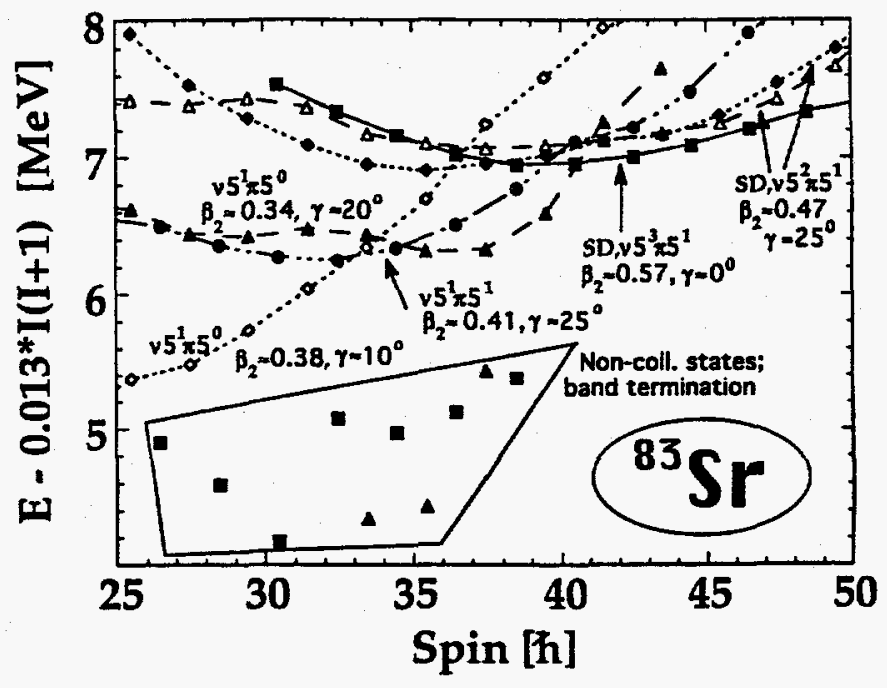

Figure 9: Predicted low-lying SD bands in ${ }^{83} \mathrm{Sr}$ [Baktash et al., 1995a].

On the other hand, some disagreements with data have also been encountered. For example, theoretical proton singie-particle routhians do not show a significant energy gap at $Z \sim 40$ which could account for the large population intensity and quadrupole moments observed in the $\mathrm{Zr}$ isotopes. Similarly, the twinned bands observed in the ${ }^{82} \mathrm{Sr}_{-}{ }^{83} \mathrm{Y}$ and ${ }^{83} \mathrm{Sr}^{84}{ }^{84} \mathrm{Y}$ isotones can be explained only for a large deformation of $\beta_{2} \sim 0.58$, which is in disagreement with the measured transition moments. It seems that some modifications of the single-particle energy spectra are called for to accommodate these experimental observations.

In general, results of other models [e.g., Aberg et al., 1995; Afanasjev et al., 1996] share many similarities with these calculations, but they also differ in some details. For example, while cranked relativistic mean field calculations by Afanasjev et al. [1996] for the SD band in ${ }^{83} \mathrm{Sr}$ predict the same $v 5^{3} \pi 5^{1}$ configuration, they calculate a larger equilibrium deformation $\left(\beta_{2} \sim 0.65\right.$ vs. 0.57$)$. Future lifetime measurements using the completed Gammasphere array will be able to distinguish between these models.

\section{SYSTEMATICS OF THE EVEN-EVEN $\mathrm{T}_{\mathrm{z}}=1$ NUCLEI AND THE QUESTION OF $\mathrm{T}=0$ np-PAIRING CORRELATIONS}

One of the most challenging and important nuclear structure questions to be investigated in the next few years is if and when the isoscalar $\mathrm{T}=0 n p$ pairing correlations become dominant in the structure of $N \sim Z$ mediummass nuclei. Although this topic was theoretically studied in the sixties and seventies (see, e.g., the review article by Goodman. 1979), paucity of experimental data has made it difficult to subject these calculations to a 
meaningful test. Thus the renewal of interest in this subject had to wait until extensive spectroscopic studied of $\mathrm{N} \sim \mathrm{Z}$ nuclei became possible. Recently, the advent of new and powerful detector systems on one hand, and development of accelerated radioactive ion beams on the other hand have largely diminished the obstacles that had impeded these experimental studies. As a result, we are now witnessing a resurgence in the theoretical investigations of various aspects of the $n p$ pairing field such as the necessary conditions for its emergence, its signature, and different theoretical approaches that would allow its incorporation in realistic calculations. Among the more recent studies are the Shell Model Monte Carlo calculations of the strengths of the pairing correlations and their temperature dependence in $N \sim Z$ nuclei of the $p f$ shell by Laganke et al. [1996]; a cranked mean-field model that includes the two-body $T=1$ and $T=0$ pairing interactions to study the high-spin behavior of these two competing forces by Satula and Wyss [1996b]; and several detailed examinations of the stability and applicability of quasiparticle random phase approximation (QRPA) and renormalized QRPA to calculate the single- and double-beta decay rates which are very sensitive to the strength of the $n p$ correlations [see, e.g., Toivanen and Suhonen, 1995; Schwieger et al., 1996; Hirsch et al., 1996; Cheoun et al, 1993; Dukelsky and Schuck, 1996; and references therein ]. Last, but not least, exactly solvable schematic models that conserve isospin symmetry have also been devised to isolate the effects of the isovector and isoscalar pairing and to test the validity of RQRPA and generalized BCS approximations in the region of phase transition [Engel et al., 1996a; Engel et al., 1996b].

Since the Fermi levels of neutrons and protons are far apart in nuclei with significant neutron excess, it is generally assumed that $n p$ pairing may be safely neglected. This assumption, however, is not valid in the $N \sim Z$ nuclei where, by and large, neutrons and protons occupy the same orbitals. For instance, except for ${ }^{34} \mathrm{Cl}$, the ground states of all odd-odd self-conjugate nuclei in the $s d$-shell have $\mathrm{T}=0, \mathrm{~J} \neq 0$, indicating the importance of the isoscalar $n p$ interactions. Because of their small number of nucleons, however, these nuclei do not develop a strong "pairing field". On the other hand, in $A>40$ nuclei where pairing correlations become more important, the evidence for the existence of a strong isoscalar $n p$ pairing becomes ambiguous. For example, with the exception of ${ }^{58} \mathrm{Cu}$, the odd-odd $N=Z$ nuclei from Sc to Ga have $\mathrm{T}=1, \mathrm{~J}=0$ ground states. Therefore, evidence for the importance of $T=0$ pairing should be sought in the structure of the excited states in these nuclei.

A major problem in the study of isoscalar pairing is the lack of an unambiguous signature. For example, Van Isacker et al. [1995] have recently suggested that the following function of the binding energies:

$$
1 / 4\{[\mathrm{~B}(N, Z)-\mathrm{B}(N-2, Z)]-[\mathrm{B}(N, Z-2)-\mathrm{B}(N-2, Z-2)]\},
$$

peaks at the $N=Z$ line, indicating the presence of $S U(4)$ symmetry in the self-conjugate nuclei. It has, however, been pointed out by Engel et al. [1996a] that this feature holds equally well in the pure isovectorseniority model as well. Similarly, the staggering between the masses of the self-conjugate odd-odd vs. even-even nuclei is the same in the two limits of pure isoscalar and isovector pairing [Engel et al., 1996b], removing it as a potential probe of $\mathrm{T}=0$ pairing. These authors also observe that another potential signature of the isoscalar pairing, namely the Gamow-Teller $\beta^{+}$strength, is also indistinguishable in the two limiting cases in $N=Z$ nuclei. (In fact, these calculations indicate that the difference between the isovector and isoscalar $B(G T)$ becomes larger as $T_{z}$ increases and $T=0$ pairing becomes less important.)

In principle, enhanced deuteron-transfer strength is a potentially clear indicator of the presence of $T=0, \mathrm{~J}=1$ pairing strength (Nazarewicz, 1996). Unfortunately, until radioactive ion beams of medium-mass $N=Z$ nuclei become available, this probe remains inaccessible. However, the inverse process (namely, enhanced emission of deuterons from moderately-excited self-conjugate compound nuclei ) may provide some clue regarding the existence of $T=0$ pairing which is predicted by Langanke et al. [1996] to survive at finite temperatures .

Given these difficulties, we are, therefore, presently limited to seek the evidence for isoscalar pairing in the band structures of $N \sim Z$ nuclei, especially at high spins. This is because of the fact that protons and neutrons coupled to $\mathrm{T}=0, \mathrm{~J}=1$ are not susceptible to the Coriolis antipairing effects the way those coupled to $\mathrm{J}=0$ (timereversed orbitals) are. In fact, a recent study of the high-spin band structure in ${ }^{74} \mathrm{Rb}$ [Rudolph et al., 1996d] suggests that the $T=1 . J=0$ ground state band is crossed by a $T=0, J=1$ excited band at spin $\sim 3 \hbar$. Although 
extremely challenging, measurements of the g-factors of the levels in the excited band may distinguish between a simple $\mathrm{T}=0$ two-quasiparticle configuration based on [431]3/2 neutron and proton orbitals and the exotic $\mathrm{T}=0$ collective $n p$-pairing mode.

As part of a program to address these questions, we have recently initiated a systematic study of the high spin band structures of the $N \sim Z$ nuclei. The $\mathrm{T}_{z}=1$ nuclei ${ }^{74} \mathrm{Kr},{ }^{78} \mathrm{Sr},{ }^{82} \mathrm{Zr}$ [Rudolph et al., 1996b], and ${ }^{86} \mathrm{Mo}$ [Rudolph et al., 1996e] have been studied using the Gammasphere + Microball experimental setup. The 130 $\mathrm{MeV}{ }^{28} \mathrm{Si}+{ }^{58} \mathrm{Ni}$ reaction was used to populate the high spin states in ${ }^{74} \mathrm{Kr},{ }^{78} \mathrm{Sr},{ }^{82} \mathrm{Zr}$ via $3 \alpha, 2 \alpha$, and $2 p 2 n$ channels, respectively. Altogether, 15 new rotational bands were established in these three nuclei. Figures 10 and 11 show the partial level schemes developed in this work for ${ }^{74} \mathrm{Kr}$ and ${ }^{82} \mathrm{Zr}$, respectively. These results, as well as the recent high-spin data for ${ }^{74} \mathrm{Rb}$ [Rudolph et al., 1996d], ${ }^{75} \mathrm{Rb}$ [Gross et al., 1996b], ${ }^{76} \mathrm{Rb}$ [Harder et al., 1995], and ${ }^{75} \mathrm{Kr}$ [Gross et al., 1994] were interpreted using the pairing-and-deformation self-consistent cranking model of Satula and Wyss [1994]. Surprisingly, this model provides a good description of the properties of the bands in $\mathrm{Kr}, \mathrm{Rb}$, and $\mathrm{Sr}$ isotopes using the conventional $\mathrm{T}=1$ pairing channel, without any need to invoke collective $\mathrm{T}=0 n p$ correlations [Gross et al., 1996b]. In contrast, these calculations cannot provide a satisfactory interpretation of the ground state band (gsb) in ${ }^{82} \mathrm{Zr}$. Figure 12 shows a comparison of the experimental (filled symbols) and calculated (open symbols) routhians for the gsb in ${ }^{74} \mathrm{Kr}$ and ${ }^{82} \mathrm{Zr}$ nuclei, as well as one of the excited bands (proton $\mathrm{AB}$ ) in ${ }^{74} \mathrm{Kr}$. Below the band crossing, the calculated routhian for a well-deformed ground-state band (wdb) in ${ }^{82} \mathrm{Zr}$ lies higher than the experimental one by about $1.5 \mathrm{MeV}$.

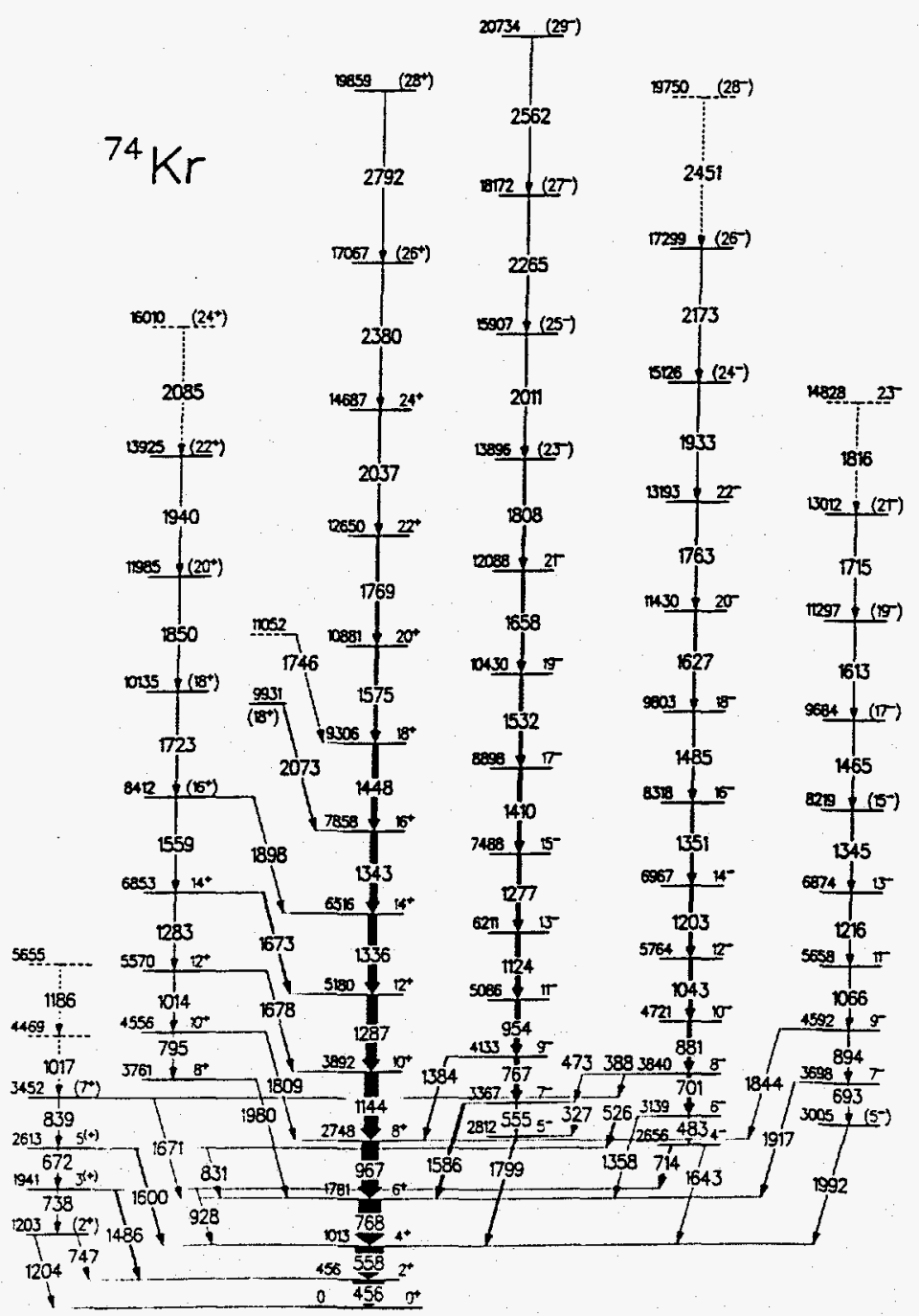

Figure 10: A partial level scheme for ${ }^{74} \mathrm{Kr}$ [Rudolph et al.. 1996b]. 


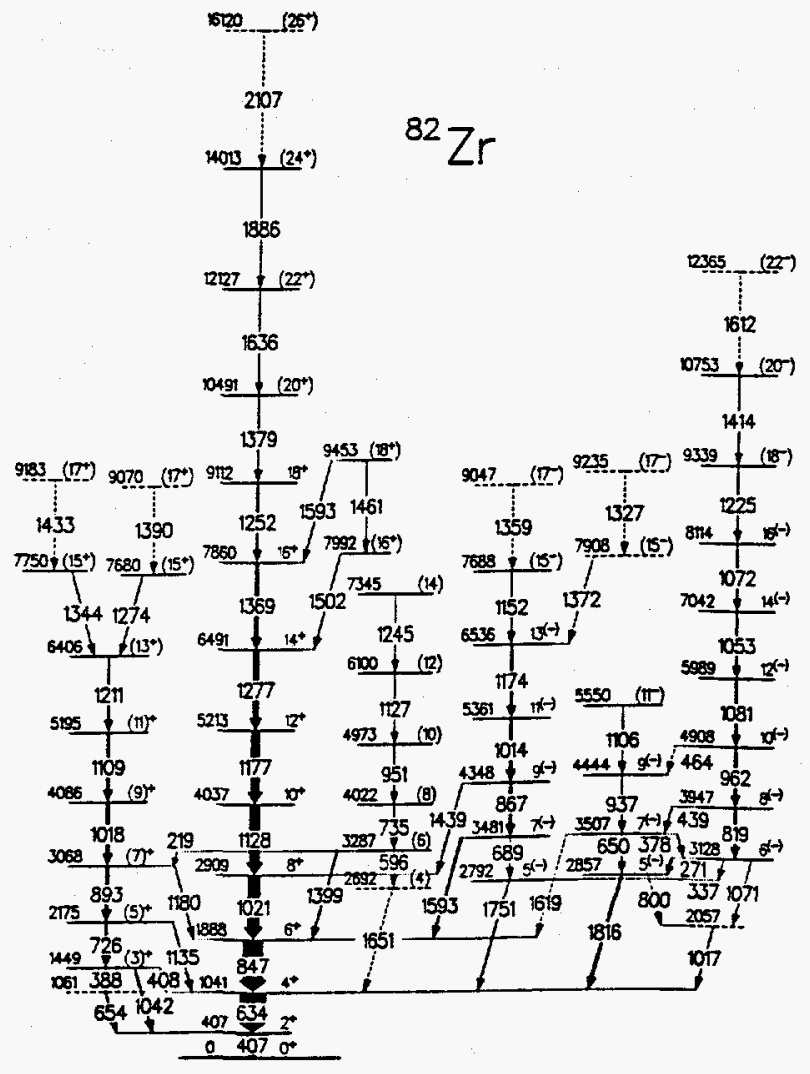

Figure 11: A partial level scheme for ${ }^{82} \mathrm{Zr}$ [Rudolph et al., 1996b].
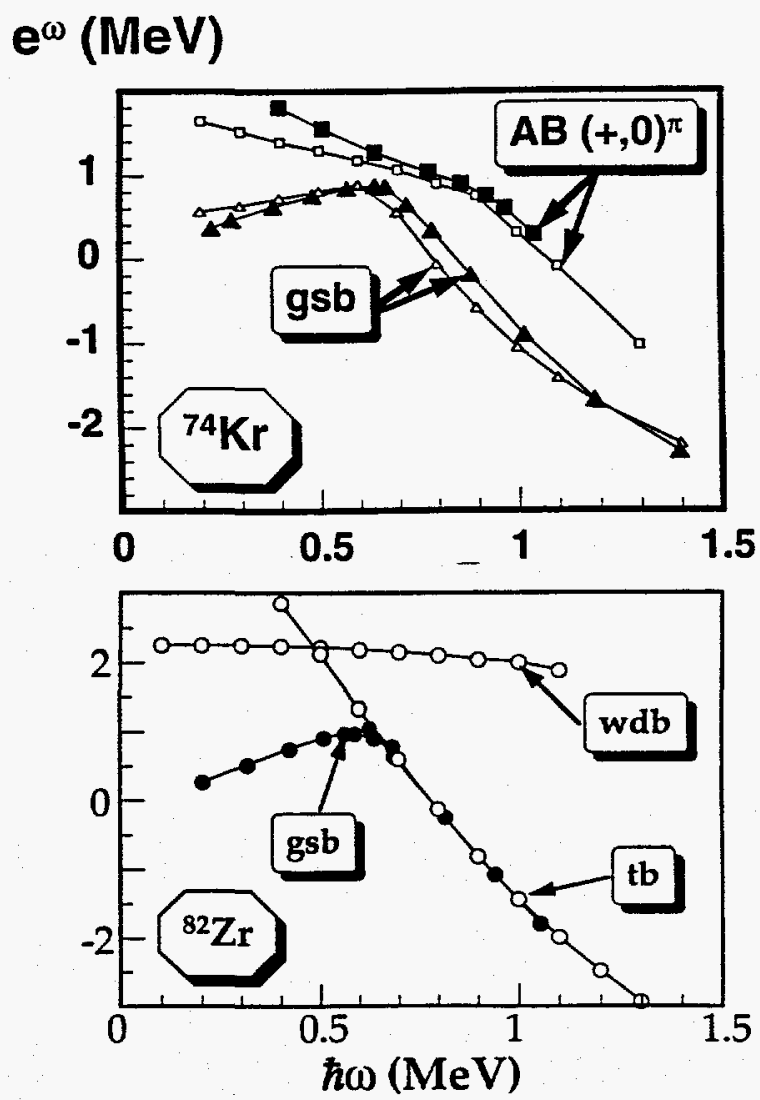

Figure 12: Comparisons of the experimental and calculated routhians for the positive-parity bands in ${ }^{74} \mathrm{Kr}$ (top) and ${ }^{82} \mathrm{Zr}$ (bottom) [Rudolph et al., 1996b]. 
This failure has been attributed to the softness of the total routhian surfaces in ${ }^{82} \mathrm{Zr}$ at low rotational frequencies which give rise to vibrational degrees of freedom. These vibrational effects cannot be accounted for in the present version of the model. In contrast, the calculated triaxial band (tb) which becomes yrast above the band crossing is in excellent agreement with the experimental routhian.

In short, these data do not provide any compelling evidence for the presence of collective $n p$ correlations in $N \sim Z$ nuclei below $\mathrm{Zr}$. They do, however, provide a warning that interpretation of these shape-transitional nuclei requires a great deal of care. That is, to isolate the effects of the isoscalar pairing field one needs to employ sophisticated models that can correctly account for all aspects of the mean field, both deformation and the pairing degrees of freedom.

\section{SUMMARY AND OUTLOOK}

Development of the new generation of powerful $\gamma$-ray detector systems has greatly facilitated spectroscopic studies of the high-spin states in the medium-mass nuclei. For example, a new region of SD nuclei in the mass A $\sim 80$ was recently discovered and extensive data on the band structures of the $N \sim Z$ nuclei have been obtained. These data may be used to test different theoretical models or to constrain their parameters.

So far, more than 20 SD bands have been observed in 10 nuclei which span $N=42-46$ and $Z=38-41$, thus confirming the earlier predictions for the existence of a large SD gap at $N \sim 44$. In general, the properties of these bands are in good agreement with the predictions of the cranking models. However, the presence of several disagreements point to possible shortcomings in the single-particle energy spectra at large deformations and rotational frequencies. The high rotational frequencies sustained by these bands correspond to the largest Coriolis force encountered so far, and allow for the observation of the $i_{13 / 2}$ superintruder orbital in these medium-mass nuclei. Another consequence of the high rotational frequency and large SD energy gaps is that the $T=1$ pairing correlations become negligibly small. This paves the way to observe the influence of the $T=0$ $p n$ interaction in the SD bands of the $N=Z \sim 30$ nuclei which are yet to be discovered. Finally, because of the dominance of charged-particle emission in this region, it is now possible to probe possible nuclear structure effects on the energies and angular distributions of the emitted protons and alpha particles. This will open up new and exciting opportunities to study nuclear structure and reaction at their interface.

The combination of the new Ge detectors and ancillary detector systems have also allowed studies of the highspin band structures of the $N \sim Z$ nuclei from $\mathrm{Kr}$ to Mo. These data provide a valuable opportunity to study the possible effects of the collective $n p$ pairing correlations that are expected to become important in these nuclei. At present, there is no conclusive evidence that the isoscalar pairing mode plays a significant role in the band structures of the $T_{z}=1 / 2$ and 1 nuclei studied so far. However, the recent high-spin data on ${ }^{74} \mathrm{Rb}$ have highlighted the need to extend the present studies to the $N=Z$ nuclei. Finally, to be able to clearly isolate the effects due to the $T=0$ pairing correlations, theoretical calculations need to be able to correctly account for the shape changes that take place in these transitional nuclei.

\section{ACKNOWLEDGMENTS}

Much of the experimental work reviewed here is the result of a large collaboration involving researchers from ORNL, Washington University, University of Pittsburgh, LBNL, and Florida State University. I am greatly indebted to the members of this collaboration group, especially Y. Akovali, M.J. Brinkamn, B. Cederwall, F. Cristancho, D.M. Cullen, J. Doring, M. Devlin, C.J. Gross, H.-Q. Jin, D.R. LaFosse, I.-Y. Lee, F. Lerma, A.O. Macchiavelli, D. Rudolph, J.X. Saladin, D.G. Sarantites, D.W. Stracener, G. Sylvan, S.L. Tabor, D.F. Winchell, and V. Wood, and C.-H. Yu. Many fruitful discussions with and theoretical support by W. Nazarewicz, W. Satula, T. Werner and R. Wyss are gratefully acknowledged. Oak Ridge National Laboratory is managed by Lockheed Martin Research Corp. for the U.S. Department of Energy under contract No. DE--AC05-96OR22464. 


\section{REFERENCES}

Abdelrazek, M., et al. (1996). To be published.

Aberg, S., H. Flocard and W. Nazarewicz (1995). Annu. Rev. Nucl. Part. Sci. 40, 439.

Afanasjev, A.V., J. Konig and P. Ring (1966). Phys. Lett. B367, 11.

Baktash, C., et al. (1995a). Phys. Rev. Lett. 74, 1946.

Baktash, C., B. Haas and W. Nazarewicz (1995b). Annu. Rev. Nucl. Part. Sci. 45, 485.

Beausang, C, et al. (1988). Nucl. Instrum. Meth. A267, 144.

Cederwall, B., et al. (1995). Nucl. Instrum. Meth. Phys. Res. A354, 591.

Cheoun, M.K., A. Faessler, F. Simkovic, G. Teneva, and A. Bobyk (1993). Nucl. Phys. A561, 74.

Christancho, F., et al. (1995). Phys. Lett. B357, 281.

Cormier, T., et al. (1983). Nucl. Instrum. Meth. 212, 185.

Cullen, D.M., et al. (1994). In Conference on Physics from Large $\gamma$-Ray Detector Arrays, Vol. I, p. 44.

Dagnall, P.J., et al. (1995). Z.Phys. A353, 251.

deFrance, G., et al. (1996). Phys. Rev. C53, R1070.

Devlin, M., et al. (1996). In: Proc. Int. Workshop on Nuclear Structure at the Limits. To be published.

Dudek, J., W. Nazarewicz and N. Rowley (1987). Phys. Rev. C35, 1489.

Dukelsky, J., and P. Schuck (1996). Phys. Lett. B387, 233.

Engel, J., K. langanke, and P. Vogel (1996a). Phys. Lett. B (in press).

Engel, J., et al. (1996b). LANL preprint nucl-th/9610045.

Ennis, P.J., et al. (1991). Nucl. Phys. A535, 392.

Gelletly, W., et al. (1991). Phys. Lett. B253, 287.

Goodman, A. L., (1979). Adv. Nucl. Phys. 11, 263.

Gross, C.J., et al. (1994). Phys. Rev. C49, R580.

Gross, C.J., et al. (1996a). In: Proc. 14th Int. Conf. on Applications of Accelerators in Research and Industry - 1996. To be published.

Gross, C.J., et al. (1996b). To be published.

Han, X.-L., and C.-L. Wu (1992). At. Data Nucl. Data Tables 2, 43.

Harder, A., et al. (1995). Phys. Rev. C51, 2932.

Hirsch, J.G., P.O. Hess, and O. Civitarese (1996). LANL preprint nucl-th/9608041.

James, A.N., et al. (1988). Nucl. Instrum. Meth. A267, 144.

Jin,H.-Q., et al. (1995). Phys. Rev. Lett. 75, 1471.

Jin, H.-Q., et al. (1996). Bull. Am. Phys. Soc. 41, 881. To be published.

Jin, H.-Q., et al. (1996b). To be published.

LaFosse, D.R., et al. (1995). Phys. Lett. B354, 34.

LaFosse, D.R., et al. (1997). Phys. Rev. Lett. (in press).

Langanke, K., et al. (1996). Nucl. Phys. A (in press).

Mantica, P., et al. (1995). Nucl. Instrum. Meth. Phys. Res. B99, 338.

Moller, P., and J.R. Nix (1981). At. Data and Nucl. Data Tables 26, 165.

Moore, E.F., et al. (1989). Phys. Rev. Lett. $\underline{63}, 360$.

Nazarewicz, W., et al. (1985). Nucl. Phys. A435, 397.

Nazarewicz, W., (1996). Private communication.

Nolan, P.J., et al. (1990). Nucl. Phys. A520, 657c.

Petrovici, A., K.W. Schmid, and A. Faessler (1996). Nucl. Phys. A605, 290.

Polikanov, S.M., et al. (1962). Sov. Phys. JETP 15, 1016.

Ragnarsson, I., S.G. Nilsson and R.K. Sheline (1978). Phys. Rep. 45, 1.

Schwieger, J., F. Simkovic, and A. Faessler, (1996). Nucl. Phys. A600, 179.

Rudolph, D., et al. (1996a). Phys. Lett. B (in press).

Rudolph, D., et al. (1996b). In: book of abstracts of Proc. Int. Workshop on Nuclear Structure at the Limits. To be published.

Rudolph, D., et al. (1996c). To be published.

Rudolph, D., et al. (1996d). Phys. Rev. Lett. 76, 376.

Rudoloh. D. tal. (1996e). Phvs. Rev. C54. 117. 
Simpson, J., et al. (1988). Nucl. Instrum. Meth. A269, 209.

Sarantites, D.G., et al. (1996). Nucl. Inst. and Meth. A (in press).

Satula, W., and R. Wyss (1994). Phys. Rev. C50, 2888.

Satula, W., et al. (1996a). Phys. Rev. Lett. 77, 5182 (1996).

Satula, W., and R. Wyss (1996b). Phys. Lett. (in press).

Smith, A.G., et al. (1995). Phys. Lett. B355, 32.

Strutinsky, V.M., (1967). Nucl. Phys. A95. 420.

Strutinsky, V.M., (1968). Nucl. Phys. A122, 1.

Toivanen, J., and J. Suhonen (1995). Phys. Rev. Lett. 75, 410.

Twin, P.J., et al. (1986). Phys. Rev. Lett. 57, 811.

Van Isacker, P., D.D. Warner, and D.S. Brenner (1995). Phys. Rev. Lett. 74, 4607.

Yu, C.-H., et al. (1996). To be published.

\section{DISCLAIMER}

This report was prepared as an account of work sponsored by an agency of the United States Government. Neither the United States Government nor any agency thereof, nor any of their employees, makes any warranty, express or implied, or assumes any legal liability or responsibility for the accuracy, completeness, or usefulness of any information, apparatus, product, or process disclosed or represents that its use would not infringe privately owned rights. Reference herein to any specific commercial product, process, or service by trade name, trademark, mot necessarily constitute or imply its endorsement, recomor favoring by the United States Government or any agency thereof. The views mendation, or favoring by the and opinions of authors expressed herein 